\title{
Association of the insulin resistance marker TyG index with the severity and mortality of COVID-19
}

Huihui Ren, Yan Yang, Fen Wang, Yongli Yan, Xiaoli Shi, Kun Dong, Xuefeng Yu and Shujun Zhang*

\begin{abstract}
Background: The triglyceride and glucose index (TyG) has been proposed as a marker of insulin resistance. This study aims to evaluate the association of the TyG index with the severity and mortality of coronavirus disease 2019 (COVID-19).

Methods: The study included a cohort of 151 patients with COVID-19 admitted in a tertiary teaching hospital in Wuhan. Regression models were used to investigate the association between TyG with severity and mortality of COVID-19.

Results: In this cohort, 39 (25.8\%) patients had diabetes, 62 (41.1\%) patients were severe cases, while 33 (22.0\%) patients died in hospital. The TyG index levels were significantly higher in the severe cases and death group (mild vs. severe $8.7 \pm 0.6$ vs. $9.2 \pm 0.6, P<0.001$; survivor vs. deceased $8.8 \pm 0.6$ vs. $9.3 \pm 0.7, P<0.001$ ), respectively. The TyG index was significantly associated with an increased risk of severe case and mortality, after controlling for potential confounders (OR for severe case, 2.9, 95\% Cl 1.2-6.3, $P=0.007$; OR for mortality, 2.9, 95\% Cl 1.2-6.7, $P=0.016$ ). The associations were not statistically significant for further adjustment of inflammatory factors.
\end{abstract}

Conclusion: TyG index was closely associated with the severity and morbidity in COVID-19 patients, thus it may be a valuable marker for identifying poor outcome of COVID-19.

Keywords: TyG index, COVID-19, Mortality, Severity

\section{Background}

In December 2019, an outbreak of pneumonia caused by the 2019 novel coronavirus disease was first observed in Wuhan, China [1]. On Feb 11 2020, the World Health Organization (WHO) officially named the disease as Coronavirus Disease 2019 (COVID-19). By Feb 19, 2020, a total of 74283 cases with COVID-19 in China have been reported, with 2009 (2.7\%) death [2]. Although most patients with COVID-19 have mild symptoms and good prognosis [3], accumulating evidence have indicated a

*Correspondence: sjzhang0407@163.com

Department of Endocrinology, Tongji Hospital, Tongji Medical College, Huazhong University of Science and Technology, Wuhan 430030, Hubei, People's Republic of China high mortality of the COVID-19 patients (11\%) [1, 4], resembling that of severe acute respiratory syndrome coronavirus (SARS-CoV) infection [5]. A few patients rapidly developed severe pneumonia, acute respiratory distress syndrome, cardiac injury, or multiple organ failure and died [4]. However, data on the clinical characteristics of patients with COVID-19 are limited, and the underlying mechanism is not clear yet. Therefore, early identification of the severity of COVID-19 is very important for public health.

Recently, the triglyceride-glucose (TyG) index, a product of triglycerides and glucose, is widely used as a reliable surrogate marker of insulin resistance (IR) [6]. Studies have shown that the TyG index is associated with an increased risk of diabetes [7], hypertension [8],

(c) The Author(s) 2020. This article is licensed under a Creative Commons Attribution 4.0 International License, which permits use, sharing, adaptation, distribution and reproduction in any medium or format, as long as you give appropriate credit to the original author(s) and the source, provide a link to the Creative Commons licence, and indicate if changes were made. The images or other third party material in this article are included in the article's Creative Commons licence, unless indicated otherwise in a credit line to the material. If material is not included in the article's Creative Commons licence and your intended use is not permitted by statutory regulation or exceeds the permitted use, you will need to obtain permission directly from the copyright holder. To view a copy of this licence, visit http://creativeco mmons.org/licenses/by/4.0/. The Creative Commons Public Domain Dedication waiver (http://creativecommons.org/publicdomain/ zero/1.0/) applies to the data made available in this article, unless otherwise stated in a credit line to the data. 
nonalcoholic fatty liver disease [9], and might predict the development of cardiovascular events [10]. Patients with cardiometabolic disorders may be more susceptible to infection of COVID-19 and becoming severe case, partly due to their abnormal metabolism and systemic inflammation state. Thus, these patients might have a higher risk of poor outcome. In this sense, studies that evaluate the relationship between TyG index with the severity and mortality of COVID-19 are urgently needed.

In this study, we investigated patients with confirmed COVID-19 who were admitted to Wuhan Tongji hospital. The purpose of this study was to explore the association of TyG index levels with the clinical outcomes of patients with COVID-19 and to provide ideas for improving COVID-19 prognosis.

\section{Methods}

\section{Study population}

This study retrospectively analysed confirmed COVID19 from Jan 12 2020, to Feb 132020 at Tongji Hospital of Huazhong University of Science and Technology in Wuhan, China. All patients enrolled in this study were diagnosed as COVID-19 according to WHO interim guidance [11]. Patients with missing data on clinical characteristics were excluded. Considering the complexity of the disease, as well as the uncertainty of the course of treatment, 151 patients which had completed medical records and follow up data were included. This study was approved by the Ethics Commission of Tongji Hospital, and all participants provided oral informed consent.

\section{Data collection}

We reviewed the epidemiological, demographic, clinical, and laboratory data from patients' medical records. Two researchers checked the data collected independently. We collected information on socioeconomic characteristics (age, sex, exposure history), smoking, chronic medical histories (diabetes, hypertension, cardiovascular disease, cerebrovascular disease, chronic kidney disease, chronic pulmonary disease, chronic liver disease), clinical symptoms (fever, cough, expectoration, dyspnoea, chest pain, diarrhea, headache, hypodynamia, anorexia, nausea and vomiting), vital signs (temperature, heart rate, respiratory rate, oxygen saturation, blood pressure), laboratory data (complete blood count, liver and renal function, fasting plasma glucose (FPG), hemoglobin A1c (HbA1c), lipid, high-sensitivity C-reactive protein (CRP), Ferritin, and tumor necrosis factor $\alpha(\mathrm{TNF} \alpha)$ ), as well as living status during study period.

\section{Definitions}

The TyG index was calculated by the formula ln [fasting triglycerides $(\mathrm{mg} / \mathrm{dL}) \times$ FPG $(\mathrm{mg} / \mathrm{dL}) / 2]$. The patients were divided into 3 tertiles according to TyG index levels, T1 $(\mathrm{n}=50,7.5 \leq$ TyG index $\leq 8.6)$, T2 $(\mathrm{n}=51,8.7 \leq$ TyG index $\leq 9.1)$, T3 $(n=50,9.2 \leq$ TyG index $\leq 10.7)$. Diabetes was defined as self-reported medical history of diabetes, and the use of antidiabetic drugs. Hypertension was defined as self-reported history of hypertension, and the use of antihypertensive drugs. Other chronic diseases, such as cardiovascular disease, cerebrovascular disease, chronic kidney disease, chronic pulmonary disease, and chronic liver disease, were diagnosed according to selfreported medical history.

Severity of the disease was staged according to the guidelines for diagnosis and treatment of COVID-19 (trial sixth edition) published by National Health Commission of China on February 18, 2020. Severe case was defined as including one criterion as follow: 1 . respiratory rate $>30 / \mathrm{min}, 2$. oxygen saturation $\leq 93 \%, 3 . \mathrm{PaO} 2 / \mathrm{FiO} 2$, 4. Patients developed either with shock, or respiratory failure requiring mechanical ventilation, or combined with the other organ failure admission to intensive care unit (ICU).

\section{Statistical analysis}

We presented continuous variables as mean \pm standard deviation (SD) for normally distributed data or median (interquartile range, IQR) for data with skew distribution, and categorical variables as frequencies (percentage, \%). We assessed differences between mild and severe cases using two-sample $T$ test or Mann-Whitney $U$ test depending on parametric or nonparametric data for continuous variables and Chi square test for categorical variables. Comparisons among TyG index groups were assessed by the analysis of variance (ANOVA) or Kruskal-Wallis test. Univariate and multivariate logistic regression analyses were used to evaluate the associations between TyG index and the severity and mortality of COVID-19. The associations of TyG index with severity of COVID-19 in subgroups classified by age $(<60$ vs. $\geq 60$ years), sex (male vs. female), and medical history of chronic disease (presence vs. absence), including diabetes mellitus and hypertension, were also studied using logistic regression analysis. We used SPSS software, version 19.0 (SPSS, Inc., Chicago, IL, USA) for all analyses with statistically significant for $P<0.05$.

\section{Results}

A total of 151 admitted hospital patients were included in our study, with a mean age of $59.5 \pm 15.9$ years old. $78(51.7 \%)$ of patients were males. Regarding the history of chronic diseases, $39.7 \%$ had hypertension, $25.8 \%$ had diabetes, $10.6 \%$ had cardiovascular disease, $4.6 \%$ had cerebrovascular disease, $7.3 \%$ had chronic kidney disease, $1.3 \%$ had chronic pulmonary disease, and $2.0 \%$ 
had chronic liver disease. The most prevalent symptoms were fever $(88.7 \%)$, cough $(68.8 \%)$, and fatigue (56.3\%). In this population, $62(41.1 \%)$ patients were severe cases, while $33(22.0 \%)$ patients died in hospital. The baseline characteristics of patients according to severity were shown in Table 1 . Severe patients were older, more males, and more likely to have hypertension and diabetes compared with mild patients. The prevalence of dyspnea, anorexia, and fatigue of the severe group were higher than that of the mild group (all $P<0.05$ ). Moreover, severe patients had significantly higher value of respiratory rate, systemic blood pressure (SBP), and mortality and/or more likely to receive auxiliary ventilation, and invasive mechanical ventilation (all $P<0.05)$, but there was no markedly difference for other parameters.

Laboratory findings of patients according to the severity were shown in Table 2 . For blood routine examination, severe patients had obviously higher values of white blood cell (WBC) count and neutrophil count (NEU), but notably lower levels of lymphocytes count than mild patients $(P<0.05)$. Regarding blood biochemical indicators, severe group had higher levels of aspartate aminotransferase (AST), lactate dehydrogenase (LDH), creatinine, uric acid (UA), CRP, and FPG, compared with mild group $(P<0.05)$. In addition, the levels of Ferritin and TNF $\alpha$ were also elevated in severe ones $(P<0.05)$. Notably, TyG index, which calculated by triglycerides and

Table 1 The basic clinical features of the COVID-19 patients according to the severity

\begin{tabular}{|c|c|c|c|c|}
\hline & Total $(\mathrm{N}=151)$ & Mild cases $(\mathrm{N}=89)$ & Severe cases $N=62$ ) & $P$ \\
\hline Age, mean $\pm S D$, years & $59.5 \pm 15.9$ & $53.9 \pm 16.2$ & $67.6 \pm 11.6$ & 0.000 \\
\hline \multicolumn{5}{|l|}{ Sex, No. (\%) } \\
\hline Male & $78(51.7 \%)$ & $38(42.7 \%)$ & $40(64.5 \%)$ & \multirow[t]{2}{*}{0.008} \\
\hline Female & $73(48.3 \%)$ & $51(57.3 \%)$ & $22(35.5 \%)$ & \\
\hline \multicolumn{5}{|l|}{ Comorbidities, No. (\%) } \\
\hline Diabetes & $39(25.8 \%)$ & $16(18.0 \%)$ & $23(37.1 \%)$ & 0.008 \\
\hline Hypertension & $60(39.7 \%)$ & $25(28.1 \%)$ & $35(56.5 \%)$ & 0.000 \\
\hline Cardiovascular disease & $16(10.6 \%)$ & $7(7.9 \%)$ & $9(14.5 \%)$ & 0.191 \\
\hline Cerebrovascular disease & $7(4.6 \%)$ & $2(2.2 \%)$ & $5(8.1 \%)$ & 0.094 \\
\hline Chronic kidney disease & $11(7.3 \%)$ & $5(5.6 \%)$ & $6(9.7 \%)$ & 0.345 \\
\hline Chronic pulmonary disease & $2(1.3 \%)$ & $1(1.1 \%)$ & $1(1.6 \%)$ & 0.796 \\
\hline Chronic liver disease & $3(2.0 \%)$ & $2(2.2 \%)$ & $1(1.6 \%)$ & 0.783 \\
\hline \multicolumn{5}{|l|}{ Signs and symptoms } \\
\hline Fever, No. (\%) & $134(88.7 \%)$ & 79 (88.8\%) & 55 (88.7\%) & 0.992 \\
\hline Cough, No. (\%) & $104(68.9 \%)$ & $58(65.2 \%)$ & $46(74.2 \%)$ & 0.239 \\
\hline Expectoration, №. (\%) & $61(40.4 \%)$ & $37(41.6 \%)$ & $24(38.7 \%)$ & 0.724 \\
\hline Dyspnea, No. (\%) & $78(51.7 \%)$ & $38(42.7 \%)$ & $40(64.5 \%)$ & 0.008 \\
\hline Pectoralgia, No. (\%) & $11(7.3 \%)$ & $7(7.9 \%)$ & $4(6.5 \%)$ & 0.742 \\
\hline Diarrhoea, No. (\%) & $54(35.8 \%)$ & $34(38.2 \%)$ & $20(32.3 \%)$ & 0.453 \\
\hline Nausea, No. (\%) & $24(15.9 \%)$ & $16(18.0 \%)$ & $8(12.9 \%)$ & 0.401 \\
\hline Vomiting, No. (\%) & $10(6.6 \%)$ & $6(6.7 \%)$ & $4(6.5 \%)$ & 0.944 \\
\hline Anorexia, No. (\%) & $57(37.7 \%)$ & $27(30.3 \%)$ & $30(48.4 \%)$ & 0.024 \\
\hline Headache, No. (\%) & $28(18.5 \%)$ & $14(15.7 \%)$ & $14(22.6 \%)$ & 0.287 \\
\hline Fatigue, No. (\%) & $85(56.3 \%)$ & $44(49.4 \%)$ & $41(66.1 \%)$ & 0.042 \\
\hline $\operatorname{Tmax}, \operatorname{mean} \pm \mathrm{SD},{ }^{\circ} \mathrm{C}$ & $38.5 \pm 0.7$ & $38.5 \pm 0.7$ & $38.7 \pm 0.7$ & 0.148 \\
\hline Respiratory rate, mean $\pm S D$, bpm & $23 \pm 5$ & $21 \pm 4$ & $26 \pm 6$ & 0.000 \\
\hline Heart rate, mean $\pm S D$, bpm & $92 \pm 18$ & $90 \pm 16$ & $94 \pm 20$ & 0.172 \\
\hline Systolic blood pressure, mean $\pm \mathrm{SD}, \mathrm{mmHg}$ & $133 \pm 20$ & $129 \pm 18$ & $138 \pm 20$ & 0.007 \\
\hline Diastolic blood pressure, mean $\pm \mathrm{SD}, \mathrm{mmHg}$ & $82 \pm 13$ & $82 \pm 12$ & $81 \pm 14$ & 0.531 \\
\hline Auxiliary ventilation, No. (\%) & $46(30.5 \%)$ & 0 & $46(74.2 \%)$ & 0.000 \\
\hline Invasive mechanical ventilation, №. (\%) & 15 (9.9\%) & 0 & $15(24.2 \%)$ & 0.000 \\
\hline Mortality, №. (\%) & $33(22.0 \%)$ & 0 & $33(54.1 \%)$ & 0.000 \\
\hline
\end{tabular}

$S D$ standard deviation, Tmax maximum body temperature

$P$ values indicate differences between mild and severe cases. $P<0.05$ was considered statistically significant 
Table 2 Laboratory findings of patients with COVID-19 on admission to hospital

\begin{tabular}{|c|c|c|c|c|}
\hline & Normal range & Mild cases $(\mathrm{N}=89)$ & Severe cases $(N=62)$ & $\mathbf{P}$ \\
\hline White blood cell count, $\times 10^{9} / \mathrm{L}$ & $3.5-9.5$ & $4.9(2.0)$ & $7.3(6.7)$ & 0.000 \\
\hline Neutrophil count, $\times 10^{9} / \mathrm{L}$ & $1.8-6.3$ & $3.0(2.1)$ & $5.8(7.1)$ & 0.000 \\
\hline Lymphocyte count, $\times 10^{9} / \mathrm{L}$ & $1.1-3.2$ & $1.2 \pm 0.5$ & $0.8 \pm 0.4$ & 0.000 \\
\hline Alanine aminotransferase, $U / L$ & $\leq 33$ & $28 \pm 32$ & $34 \pm 27$ & 0.266 \\
\hline Aspartate aminotransferase, $\mathrm{U} / \mathrm{L}$ & $\leq 32$ & $24.0(13.0)$ & $36.0(26.0)$ & 0.000 \\
\hline Lactate dehydrogenase, $\mathrm{U} / \mathrm{L}$ & $135-214$ & $251.0(93.0)$ & $431.5(367.0)$ & 0.000 \\
\hline Creatinine, $\mu \mathrm{mol} / \mathrm{L}$ & $45-84$ & $70.0(28.5)$ & $81.5(33.0)$ & 0.003 \\
\hline Uric acid, $\mu \mathrm{mol} / \mathrm{L}$ & $202.3-416.6$ & $248.0(103.9)$ & $264.5(168.9)$ & 0.036 \\
\hline Total cholesterol, $\mathrm{mmol} / \mathrm{L}$ & $<5.18$ & $3.8 \pm 0.7$ & $3.6 \pm 0.8$ & 0.089 \\
\hline C-reactive protein, $\mathrm{mg} / \mathrm{L}$ & $<1$ & $12.6(36.4)$ & $68.7(114.6)$ & 0.000 \\
\hline Erythrocyte sedimentation rate, $\mathrm{mm} / \mathrm{H}$ & $0-20$ & $28 \pm 22$ & $32 \pm 22$ & 0.477 \\
\hline Ferritin, ug/L & $15-150$ & $888.2 \pm 2181.7$ & $1869.1 \pm 2229.4$ & 0.027 \\
\hline $\mathrm{TNFa}, \mathrm{pg} / \mathrm{mL}$ & $<8.1$ & $7.3(3.3)$ & $9.1(6.0)$ & 0.002 \\
\hline $\mathrm{HbA} 1 \mathrm{c}, \%$ & $4-6$ & $6.0 \pm 1.5$ & $6.7 \pm 2.1$ & 0.083 \\
\hline Fasting glucose, $\mathrm{mmol} / \mathrm{L}$ & $4.11-6.55$ & $6.2(2.3)$ & $8.4(5.8)$ & 0.000 \\
\hline Triglyceride, mmol/L & $<1.7$ & $1.4 \pm 1.0$ & $1.5 \pm 0.6$ & 0.431 \\
\hline TyG & - & $8.7 \pm 0.6$ & $9.2 \pm 0.6$ & 0.000 \\
\hline
\end{tabular}

Continuous data were expressed as mean $\pm S D$ or median (interquartile range)

$P$ values indicate differences between mild and severe cases. $P<0.05$ was considered statistically significant

glucose, was markedly higher in severe cases than in mild ones $(P<0.05)$.

Table 3 showed the baseline characteristics among COVID-19 patients, divided according to TyG index tertiles. Compared to the first tertile of TyG index, patients in the highest tertile of TyG presented higher levels of WBC, NEU, AST, LDH, CRP, and ferritin, yet lower levels of lymphocytes count (all $P<0.05$ ). Most importantly, patients with increasing level of TyG index had a higher incidence of severe COVID-19 case and death $(P=0.008$ and 0.01 , respectively), as shown in Fig. 1.

Table 3 Characteristics of the COVID-19 patients according to the tertiles of TyG

\begin{tabular}{|c|c|c|c|c|}
\hline & \multicolumn{3}{|l|}{ TyG tertiles } & \multirow[t]{2}{*}{$P$} \\
\hline & $\mathrm{T} 1(7.5-8.6)(\mathrm{N}=50)$ & $\mathrm{T} 2(8.7-9.1)(\mathrm{N}=51)$ & T3 $(9.2-10.7)(\mathrm{N}=50)$ & \\
\hline Age, years & $53(32)$ & $66(22)$ & $67.5(18)$ & 0.006 \\
\hline Male, No. (\%) & $21(42 \%)$ & $25(49 \%)$ & $32(64 \%)$ & 0.080 \\
\hline White blood Cell count, $\times 10^{9} / \mathrm{L}$ & $4.5(1.9)$ & $5.5(4.0)$ & $6.5(4.7)$ & 0.000 \\
\hline Neutrophil count, $\times 10^{9} / \mathrm{L}$ & $2.7(1.5)$ & $4.0(4.6)$ & $4.6(5.0)$ & 0.000 \\
\hline Lymphocyte count, $\times 10^{9} / \mathrm{L}$ & $1.2 \pm 0.5$ & $1.0 \pm 0.6$ & $0.9 \pm 0.6$ & 0.018 \\
\hline Aspartate aminotransferase, $\mathrm{U} / \mathrm{L}$ & $23(18)$ & $29(13)$ & $33.5(29)$ & 0.016 \\
\hline Lactate dehydrogenase, U/L & $240.0(130.0)$ & $293.0(176.0)$ & $332.0(245.0)$ & 0.000 \\
\hline Creatinine, $\mu \mathrm{mol} / \mathrm{L}$ & $73.1 \pm 27.6$ & $78.8 \pm 31.6$ & $86.9 \pm 34.3$ & 0.089 \\
\hline Uric acid, $\mu \mathrm{mol} / \mathrm{L}$ & $260.2 \pm 80.6$ & $266.3 \pm 122.4$ & $287.2 \pm 129.2$ & 0.455 \\
\hline C-reactive protein, mg/L & $11.6(37.9)$ & $39.8(70.3)$ & $37.9(127.6)$ & 0.005 \\
\hline Ferritin, ug/L & $377.8(470.4)$ & $642.7(1247.2)$ & $1008.5(1154.1)$ & 0.000 \\
\hline $\mathrm{TNFa}, \mathrm{pg} / \mathrm{mL}$ & $7.4(2.2)$ & $8.8(5.5)$ & $9.4(6.3)$ & 0.012 \\
\hline Fasting glucose, $\mathrm{mmol} / \mathrm{L}$ & $5.5(1.2)$ & $6.5(2.1)$ & $10.8(7.8)$ & 0.000 \\
\hline Triglyceride, mmol/L & $0.9(0.3)$ & $1.4(0.5)$ & $1.8(1.0)$ & 0.000 \\
\hline TyG & $8.3(0.4)$ & $8.9(0.3)$ & $9.6(0.6)$ & 0.000 \\
\hline
\end{tabular}

Continuous data were expressed as mean $\pm \mathrm{SD}$ or median (interquartile range)

$P$ values indicate differences among the tertiles of TyG. $P<0.05$ was considered statistically significant 

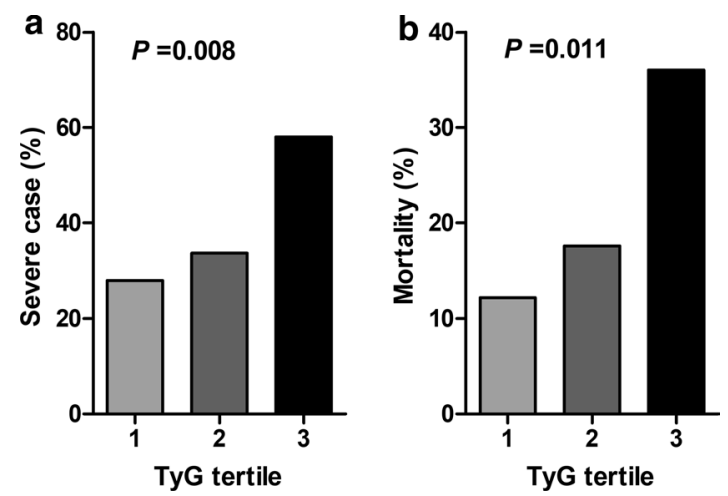

Fig. 1 Frequency of severe cases $\mathbf{a}$ and death $\mathbf{b}$ of COVID-19 patients according to the tertiles of TyG index

Additionally, the association between TyG index and the severity of COVID-19 were further confirmed by logistic regression (Table 4). After adjustment for age, sex, SBP and HbA1C, the TyG index was independently associated with the severity of COVID-19 patients with an odds ratio (OR) of 2.9 [95\% confidence interval (CI) 1.26-6.3]. However, no significant association was observed after further adjusting for CRP and TNF $\alpha$ (OR 2.3, 95\% CI 0.6-8.3).

Next, we studied correlations between TyG with CRP and TNF $\alpha$ by using Pearson correlation analysis. We observed that after controlling for age, sex, HbA1c, and
SBP, TyG levels positively correlated with both CRP $(r=0.286, P=0.003)$ and TNF $\alpha(r=0.42, P<0.0001)$.

We further assessed the effect of TyG index in particular subsets of patients with COVID-19 (Table 4). Similar results were observed in subgroups analysis. After adjustment for age, sex, SBP and HbA1C, the positively association between TyG index and the severity was observed in subgroups of men, women, age $\geq 60$, and patients with diabetes (OR 5.7, 95\% CI 1.7-19.2; OR 3.3, 95\% CI 1.5-7.0; OR 3.0, 95\% CI 1.2-7.3; OR 6.8, 95\% CI 1.3-34.8). However, the adjusted association was not statistically significant observed after adding CRP and TNF $\alpha$ in model 3 (OR 3.2, 95\% CI 0.4-22.7; OR 2.6, 95\% CI 0.8-9.0; OR 5.4, 95\% CI 51.0-30.6; OR 6.0, 95\% CI $0.7-51.7)$. Additionally, the associations were not significant in patients with age $<60$ years, or without diabetes or hypertension.

Moreover, the results showed the TyG index level was significantly higher in the death group (survivor vs. deceased $8.8 \pm 0.6$ vs. $9.3 \pm 0.7, P<0.001$ ). We used a logistic regression model to evaluate the association of TyG index with the mortality of COVID-19, and found that the TyG index was a risk factor for increased mortality of COVID-19 patients (OR 2.9, 95\% CI 1.2-6.7) after adjustment for age, sex, SBP and HbA1C (Table 5). Notably, the association was not statistically significant observed after additionally adjustment for CRP and TNF $\alpha$ (OR 2.8, 95\% CI 0.7-11.3).

Table 4 Odds ratios for severe cases associated with TyG in whole population and in different subgroups

\begin{tabular}{|c|c|c|c|c|c|c|}
\hline & \multicolumn{2}{|l|}{ Model1 } & \multicolumn{2}{|l|}{ Model2 } & \multicolumn{2}{|l|}{ Model3 } \\
\hline & OR $(95 \% \mathrm{Cl})$ & $\mathbf{P}$ & OR $(95 \% \mathrm{Cl})$ & $\mathbf{P}$ & OR (95\% Cl) & $\mathbf{P}$ \\
\hline Total & $2.8(1.6-4.8)$ & 0.000 & $2.9(1.2-6.3)$ & 0.007 & $2.3(0.6-8.3)$ & 0.207 \\
\hline \multicolumn{7}{|l|}{ Subgroups } \\
\hline \multicolumn{7}{|l|}{ Sex } \\
\hline Male & $3.6(1.6-8.2)$ & 0.002 & $5.7(1.7-19.2)$ & 0.005 & $3.2(0.4-22.7)$ & 0.246 \\
\hline Female & $2.8(1.6-4.8)$ & 0.000 & $3.3(1.5-7.0)$ & 0.003 & $2.6(0.8-9.0)$ & 0.132 \\
\hline \multicolumn{7}{|l|}{ Age } \\
\hline$\geq 60$ years & $2.6(1.2-5.7)$ & 0.013 & $3.0(1.2-7.3)$ & 0.014 & $5.4(1.0-30.6)$ & 0.055 \\
\hline$<60$ years & $1.8(0.7-4.9)$ & 0.246 & $1.3(0.2-8.5)$ & 0.773 & $0.6(0.1-9.7)$ & 0.746 \\
\hline \multicolumn{7}{|l|}{ DM } \\
\hline Yes & $6.5(1.4-29.6)$ & 0.016 & $6.8(1.3-34.8)$ & 0.021 & $6.0(0.7-51.7)$ & 0.103 \\
\hline No & $1.9(0.9-3.7)$ & 0.077 & $1.2(0.6-2.7)$ & 0.630 & $1.2(0.4-4.0)$ & 0.780 \\
\hline \multicolumn{7}{|l|}{ Hypertension } \\
\hline Yes & $4.6(1.6-13.3)$ & 0.006 & $4.3(1.0-18.7)$ & 0.054 & $6.3(0.6-64.2)$ & 0.121 \\
\hline No & $1.7(0.8-3.5)$ & 0.138 & $1.8(0.6-5.3)$ & 0.269 & $1.7(0.3-8.6)$ & 0.520 \\
\hline
\end{tabular}

Model1: unadjusted

Model2: adjusted for age, sex, $\mathrm{HbA1C}$, and SBP

Model3: adjusted for age, sex, SBP, HbA1C, CRP, and TNFa

$O R$ odds ratio, $C l$ confidence interval, $D M$ diabetes mellitus, SBP systolic blood pressure, $C R P C$-reactive protein 
Table 5 Association of TyG and mortality in total population

\begin{tabular}{llll}
\hline & Survival & Death & $\mathbf{P}$ \\
\hline TyG levels & $8.8 \pm 0.6$ & $9.3 \pm 0.7$ & 0.000 \\
OR $(95 \% \mathrm{Cl})$ & & & \\
Model1 & 1 & $3.0(1.6-5.6)$ & 0.001 \\
Model2 & 1 & $2.9(1.2-6.7)$ & 0.016 \\
Model3 & 1 & $2.8(0.7-11.3)$ & 0.153 \\
\hline
\end{tabular}

Model1: unadjusted

Model2: adjusted for age, sex, SBP and $\mathrm{HbA1C}$

Model3: adjusted for age, sex, SBP, HbA1C, CRP, and TNFa

$O R$ odds ratio, $C l$ confidence interval, $S B P$ systolic blood pressure, $C R P C$-reactive protein

Finally, we performed the receiver operating characteristic (ROC) curve analysis to test the ability of TyG to predict the severity and mortality of COVID-19. The optimal cut-off value of TyG was derived from the point with the maximum Youden index. The area under the ROC curve (AUROC) of TyG for predicting severe COVID-19 patients was 0.664 (95\% CI $0.576-0.751)(P=0.001)$. The optimal cut-off point of TyG was 8.5, with a sensitivity of $87.1 \%$ and specificity of $38.2 \%$. Moreover, the AUROC of TyG for predicting the mortality of COVID-19 was 0.687 (95\%CI 0.584-0.790) $(P=0.001)$. The optimal cut-off point of TyG was 9.6, yielding sensitivity and specificity of $39.4 \%$ and $92.3 \%$, respectively.

\section{Discussion}

In this study, we evaluated the role of TyG index in identifying severe cases and mortality of COVID-19 patients. We demonstrated for the first time that the incidence of severe COVID-19 case was higher among patients with increasing level of TyG index. After adjusting confounding factors, the TyG index was closely associated with an increased risk of severe patients with COVID-19. In addition, TyG index was also a valuable predictor for mortality of COVID-19.

\section{TyG index and the severity and mortality of COVID-19}

Epidemiological evidence have shown a high mortality and morbidity of diabetic patients who develop COVID19 [4]. Our data also observed a high prevalence of diabetes in COVID-19 patients. Notably, we found that the TyG index, a reliable surrogate marker of IR, were predictive of severe cases and mortality of COVID-19 patients. Previous studies have shown that the TyG index may predict the development of type 2 diabetes mellitus [12] and metabolic syndrome [13]. It has been reported that the TyG index is positively associated with the higher prevalence of subclinical coronary artery disease (CAD) [14], symptomatic $\mathrm{CAD}$ and metabolic and behavioral risk factors [15], and arterial stiffness and nephric microvascular damage [16]. Moreover, higher TyG index correlated with increased risk of main adverse cardiovascular and cerebrovascular events (MACCE) in acute ST-elevation myocardial infarction (STEMI) patients, and the TyG index might be a valuable predictor of clinical outcomes in STEMI patients undergoing percutaneous coronary intervention [17]. Moreover, an elevated TyG index was significantly associated with a higher risk of arterial stiffness and nephric microvascular damage. The positive association of TyG index with increased risk of developing severe cases and the risk of mortality in confirmed COVID-19 patients, could be attributed to the roles of IR in metabolic disorder, an altered inflammation profile, and cardiovascular disease risk factors. These findings support the significance of TyG index as a valuable predictor of poorer outcomes for COVID-19 patients, and remind physicians that more intensive attention should be paid to patients with high level of TyG index.

\section{Inflammation and the severity and mortality of COVID-19}

The virus of COVID-19 was classified as beta-coronavirus, and had highly pathogenicity similar to SARS$\mathrm{CoV}$ and middle east respiratory syndrome coronavirus (MERS-CoV), that could cause severe respiratory syndrome in humans [18-20]. Yet, the fundamental pathophysiology of COVID-19 remains unknown. Previous studies have shown that increased concentrations of proinflammatory cytokines in serum (e.g., IL1B, IL6, IL12, IFN $\gamma$, IL10, and TNF $\alpha$ ) were induced in patients with SARS [21] and MERS [22]. Considering the large amount of cytokines induced by SARS-CoV and MERS-CoV, a cytokine storm in the body might play a crucial role in pulmonary inflammation and extensive lung damage of patients with COVID-19. In early studies, COVID-19 was also reported to induce increased secretion of T-helper-2 (Th2) cytokines (e.g., IL4 and IL10) that suppress inflammation [4]. We noted that severe patients of COVID-19 had high amounts of inflammatory indicators (Ferritin and $\mathrm{TNF} \alpha$ ) in severe patients than mild ones, suggesting that the cytokine storm was associated with disease severity.

\section{Other potential factors and the severity and mortality of COVID-19}

Consistent with previous reported, older patients were more susceptible to COVID-19, and 70\% of infected patients were men [1]. In the present study, similar results were observed in age, yet there is no difference in the prevalence of male or female in total patients with COVID-19. Notably, the present study also emphasized a greater number of men among severe cases compared 
with mild cases. These findings suggest that older, male patients was more likely to have a worse prognosis. In addition to the elderly and males, we found that patients with a history of diabetes and hypertension are at increased risk of severe COVID-19 and mortality, in agreement with previous studies [4, 23]. Nevertheless, we observed a higher prevalence of diabetes and hypertension than that reported in the above study. The high prevalence of comorbid conditions may be expected for Tongji Hospital being the designated hospital for severe cases.

We found TyG index was closely associated with the mortality of COVID-19, which may be useful to predict the poor outcome for the infected patients. Although we attempted to adjust for many confounders, it is difficult to assess the independently cause of death among the 33 dead cases. The causes of death may be respiratory failure, heart failure, inflammation storm, sepsis, disseminated intravascular coagulation, multiple organ failure, acute kidney injury, malignant arrhythmia, diabetic ketoacidosis, even sudden death, and other uncertain events. Taken together, it is not easy to identify the relationship between TyG index and any cause of death in the world.

\section{Study strengths and limitations}

This study has several strengths. First, the COVID-19 was first observed in Wuhan China. A large number of patients are suffering with COVID-19 in this city, which provided us an opportunity to evaluate the epidemic affair. Second, we benefited from obtaining accurate and thorough clinical data of patients in Tongji Hospital, which is a designated hospital responsible for the treatments for moderate-severe COVID-19 patients in Wuhan. Third, all authors are first line doctors, who could directly observe participants.

Several limitations in this study should also be addressed. First, considering the complexity of the disease, as well as the limited information on the course of disease, the number of samples is relatively small. Second, compared with published paper, the percentage of severe cases was high in our study. It may be explained by that Tongji Hospital is the designated hospital for moderate-severe cases. Third, most people were hospitalized for treatment of pneumonia, resulting that metabolic disorders were easily overlooked. Thus, we could not observe the association of metabolic control with the prognosis of COVID-19. Fourth, the TyG index, which predicted the death of COVID-19 in this study, might be affected by the change of triglycerides and glucose levels. Other factors, such as inflammation, should also be taken into consideration when predicting the death of a patient.

\section{Conclusion}

In summary, our study illustrated that the TyG index was notably high in severe and dead patients of COVID19. Moreover, the study unveiled that TyG index was an independent predictor of an increased risk of poor outcome in patients with COVID-19. These findings also highlighted the high risk of severe condition and death among patients with metabolic disorders. Despite this, understanding the mechanisms underlying the interaction of the TyG index and COVID-19 will allow for drug development aimed at treatment and/or preventing severe condition.

\section{Abbreviations}

COVID-19: Corona virus disease 2019; SARS-CoV: Severe acute respiratory syndrome coronavirus; TyG: The triglyceride-glucose index; IR: Insulin resistance; FPG: Fasting plasma glucose; HbA1c: Hemoglobin A1c; CRP: C-reactive protein; TNFa: Tumor necrosis factor a; ICU: Intensive care unit; SD: Standard deviation; IQR: Interquartile range; ANOVA: Analysis of variance; SBP: Systemic blood pressure; WBC: White blood cell; NEU: Neutrophil count; AST: Aspartate aminotransferase; LDH: Lactate dehydrogenase; UA: Uric acid; OR: Odds ratio; $\mathrm{Cl}$ : Confidence interval; ROC: Receiver operating characteristic curve; AUROC: Area under the receiver operating characteristic curve; CAD: Coronary artery disease; MACCE: Main adverse cardiovascular and cerebrovascular events; STEMI: ST-elevation myocardial infarction; MERS-CoV: Middle east respiratory syndrome coronavirus.

\section{Acknowledgements}

We sincerely thank the participants for their willingness to participates in this research study, and to the staff who carried out the extensive clinical and nursing work in Tongji Hospital.

\section{Authors' contributions}

$S Z, Y Y, X Y$, and $H R$ conceived the research and participated in the study design. HR, FW, YY, XS, GY and KD assisted in collecting the data. SZ, YY, and KD analyzed the data. SZ, HR, XS, and FW drafted the manuscript. All authors read and approved the final manuscript.

Funding

Not applicable.

\section{Availability of data and materials}

The datasets generated and analysed for this study are available from the corresponding author upon reasonable request.

\section{Ethics approval and consent to participate}

This study was approved by the Ethics Commission of Tongji Hospital, and all participants were informed and agreed to participate in this study.

\section{Consent for publication}

Not applicable.

\section{Competing interests}

The authors declare that they have no competing interest.

Received: 7 March 2020 Accepted: 7 May 2020

Published online: 11 May 2020

References

1. Chen N, Zhou M, Dong X, et al. Epidemiological and clinical characteristics of 99 cases of 2019 novel coronavirus pneumonia in Wuhan, China: a descriptive study. Lancet. 2020;395:507-13. 
2. Yang $X, Y u Y, X u$ J et al. Clinical course and outcomes of critically ill patients with SARS-CoV-2 pneumonia in Wuhan, China: a single-centered, retrospective, observational study. Lancet Respir Med. 2020.

3. Wang C, Horby PW, Hayden FG, Gao GF. A novel coronavirus outbreak of global health concern. Lancet. 2020;395:470-3.

4. Huang C, Wang Y, Li X, et al. Clinical features of patients infected with 2019 novel coronavirus in Wuhan, China. Lancet. 2020;395:497-506.

5. WHO. Summary of probable SARS cases with onset of illness from 1 November 2002 to 31 July 2003. 2003. https://www.who.int/csr/sars/ country/table.2004_04_21/en/ Accessed 29 Feb 2020.

6. Du T, Yuan G, Zhang M, et al. Clinical usefulness of lipid ratios, visceral adiposity indicators, and the triglycerides and glucose index as risk markers of insulin resistance. Cardiovasc Diabetol. 2014;13:146.

7. Zhang M, Wang B, Liu Y, et al. Cumulative increased risk of incident type 2 diabetes mellitus with increasing triglyceride glucose index in normalweight people: the Rural Chinese Cohort Study. Cardiovasc Diabetol. 2017; 16:30.

8. Sanchez-Inigo L, Navarro-Gonzalez D, Pastrana-Delgado J, et al. Association of triglycerides and new lipid markers with the incidence of hypertension in a Spanish cohort. J Hypertens. 2016:34:1257-65.

9. Zhang S, Du T, Zhang J, et al. The triglyceride and glucose index (TyG) is an effective biomarker to identify nonalcoholic fatty liver disease. Lipids Health Dis. 2017;16:15

10. Sanchez-Inigo L, Navarro-Gonzalez D, Fernandez-Montero A, et al. The TyG index may predict the development of cardiovascular events. Eur J Clin Invest. 2016;46:189-97.

11. Saito T, Kuma A, Sugiura Y, et al. Autophagy regulates lipid metabolism through selective turnover of NCoR1. Nat Commun. 2019;10:1567.

12. Navarro-González D, Sánchez-Iñigo L, Pastrana-Delgado J, et al. Triglyceride-glucose index (TyG index) in comparison with fasting plasma glucose improved diabetes prediction in patients with normal fasting glucose: the Vascular-Metabolic CUN cohort. Prev Med. 2016;86:99-105.

13. Shin KA, Kim YJ. Usefulness of surrogate markers of body fat distribution for predicting metabolic syndrome in middle-aged and older Korean populations. Diabetes Metab Syndr Obes. 2019;12:2251-9.

14. Park GM, Cho YR, Won KB, et al. Triglyceride glucose index is a useful marker for predicting subclinical coronary artery disease in the absence of traditional risk factors. Lipids Health Dis. 2020;19:7.
15. da Silva A, Caldas APS, Hermsdorff HHM, et al. Triglyceride-glucose index is associated with symptomatic coronary artery disease in patients in secondary care. Cardiovasc Diabetol. 2019;18:89.

16. Zhao S, Yu S, Chi C, et al. Association between macro- and microvascular damage and the triglyceride glucose index in community-dwelling elderly individuals: the Northern Shanghai Study. Cardiovasc Diabetol. 2019;18:95.

17. Luo E, Wang D, Yan G, et al. High triglyceride-glucose index is associated with poor prognosis in patients with acute ST-elevation myocardial infarction after percutaneous coronary intervention. Cardiovasc Diabetol. 2019;18:150.

18. Lu R, Zhao X, Li J, et al. Genomic characterisation and epidemiology of 2019 novel coronavirus: implications for virus origins and receptor binding. Lancet. 2020;395:565-74.

19. Peiris JS, Guan Y, Yuen KY. Severe acute respiratory syndrome. Nat Med. 2004;10:S88-97.

20. Zaki AM, van Boheemen S, Bestebroer TM, et al. Isolation of a novel coronavirus from a man with pneumonia in Saudi Arabia. N Engl J Med. 2012;367:1814-20.

21. He L, Ding Y, Zhang Q, et al. Expression of elevated levels of pro-inflammatory cytokines in SARS-CoV-infected ACE2 + cells in SARS patients: relation to the acute lung injury and pathogenesis of SARS. J Pathol. 2006;210:288-97.

22. Mahallawi WH, Khabour OF, Zhang Q, et al. MERS-CoV infection in humans is associated with a pro-inflammatory Th1 and Th17 cytokine profile. Cytokine. 2018;104:8-13.

23. Wang D, Hu B, Hu C, et al. Clinical characteristics of 138 hospitalized patients with 2019 novel coronavirus-infected pneumonia in Wuhan. Jama: China; 2020.

\section{Publisher's Note}

Springer Nature remains neutral with regard to jurisdictional claims in published maps and institutional affiliations.

Ready to submit your research? Choose BMC and benefit from:

- fast, convenient online submission

- thorough peer review by experienced researchers in your field

- rapid publication on acceptance

- support for research data, including large and complex data types

- gold Open Access which fosters wider collaboration and increased citations

- maximum visibility for your research: over $100 \mathrm{M}$ website views per year

At BMC, research is always in progress.

Learn more biomedcentral.com/submissions 